\title{
Vacunas anti-neumocóccicas en adultos: actualización
}

Rodrigo Blamey

\section{Pneumococcal vaccines in adults: an update}

The burden of pneumococcal disease is high worldwide, mainly among elderly and immunosuppressed patients. Vaccines are important tools as preventive strategy for this population. There are two types of pneumococcal vaccines approved for adults, these are polysaccharide and conjugate, which are inactivated, have different serotype composition and immunogenicity. As new studies are available for pneumococcal conjugate vaccine, new recommendations have been made for its use in adults.

Key words: Streptococcus pneumoniae, pneumoccocal vaccines, conjugated vaccines, polysaccharide vaccines, pneumonia.

Palabras clave: Streptococcus pneumoniae, vacunas neumocóccicas, vacunas conjugadas, vacunas polisacáridas, neumonía.

\section{¿Es realmente importante vacunar a los adultos contra Streptococcus pneumoniae?}

$\mathrm{E}$ n el continente americano, más de $50 \%$ del total de muertes ocurre en personas mayores de 60 años y la neumonía es una de las principales causas de mortalidad, pudiendo variar entre el tercer y el primer lugar dependiendo del país. En Chile se estima una incidencia de aproximadamente 170.000 casos anuales de neumonía adquirida en la comunidad (NAC). El principal agente etiológico de NAC en adultos en todos los estudios siempre ha sido $S$. pneumoniae. Los factores de riesgo para adquirir enfermedades neumocóccicas invasoras (ENIs) son la edad mayor a 50 años, el tabaquismo, alcoholismo, enfermedades cardiovasculares y pulmonares crónicas, inmunodeficiencias y diabetes mellitus, entre otras. La mortalidad asociada a ENI también es mayor cuando se asocian algunas de estas co-morbilidades. Considerando la frecuencia con que las enfermedades neumocóccicas afectan a los adultos a partir de 50 años de edad, la alta prevalencia de co-morbilidades en este grupo etario, y la cada vez mayor proporción de adultos mayores en el país, es que las estrategias de prevención con vacunas son muy relevantes para ellos.

\section{¿Cuáles vacunas anti neumocóccicas están aprobadas actualmente para ser usadas en adultos?}

Dos diferentes vacunas han sido aprobadas para su uso en adultos:

- Pneumo $23^{\circledR}$ (PPSV-23): Por largo tiempo fue la única vacuna anti neumocóccica aprobada en este grupo etario. Corresponde a una vacuna tipo polisacárida, que contiene el antígeno capsular de 23 serotipos de S. pneumoniae.
- Prevenar-13 $3^{\circledR}$ (PCV-13): Recientemente aprobada para adultos en base a estudios de inmunogenicidad, esta vacuna es de tipo conjugada, y posee los antígenos capsulares de 13 serotipos de $S$. pneumoniae, conjugados a una proteína transportadora.

\section{¿Cuáles son las diferencias de inmunogenicidad y eficacia entre estas vacunas para adultos?}

Es importante señalar que no existe correlación establecida entre las concentraciones plasmáticas de anticuerpos y protección frente a la enfermedad causada por $S$. pneumoniae.

Respecto a PPSV-23, posee antígenos que gatillan una respuesta $\mathrm{T}$ independiente, sin efecto booster, sin efecto en la portación nasofaríngea ni efecto de rebaño*. Los estudios de inmunogenicidad sí han demostrado persistencia de IgG más allá de 5 a 10 años. Su eficacia ha sido materia de controversia, ya que los estudios han utilizado diferentes diseños, tamaños de muestras y metodologías para medir eficacia, y por lo tanto, los resultados han sido discordantes en las conclusiones. No obstante, existe una vasta experiencia con ella y su seguridad ha sido bien demostrada. Un meta-análisis del Cochrane (2013), que incluyó los estudios prospectivos randomizados más rigurosos, demostró su eficacia clínica en prevenir ENI (aproximadamente 74\%). Los estudios observacionales también han demostrado su eficacia en la mayoría, pero no en todos los reportes. Más controversial ha sido su efecto en la disminución de hospitalizaciones y mortalidad por neumonía, hospitalizaciones por enfermedades cardiovasculares y respiratorias. No obstante, existen varios estudios que avalan su costo-eficacia en Estados Unidos de América y en Europa.

*Protección indirecta de población no vacunada.
Hospital del Salvador. Unidad de Infectología. Clínica Las Condes. Santiago.

Financiamiento: no utilizó.

Conflictos de interés: - Ha recibido honorarios por presentaciones de MSD y Pfizer - Ha recibido apoyo para asistencia a cursos y congresos internacionales por parte de MSD, GSK y Pfizer.

Recibido: 9 de junio de 2014 Aceptado: 9 de julio de 2014

Correspondencia a: Rodrigo Blamey r_blameyd@yahoo.com 
- La vacuna PCV-13, induce una respuesta T dependiente, con memoria inmunológica y potencial efecto booster. $\mathrm{Su}$ inmunogenicidad es superior a la de la vacuna polisacárida, tanto en esquemas de administración única como en esquemas secuenciales (PVC13+PPSV-23), pero los estudios de eficacia clínica no tienen resultados publicados aún (Estudio CAPITA). Es importante señalar que este tipo de vacuna posee efecto en la portación nasofaríngea y por lo tanto, efecto de rebaño, el cual ha demostrado beneficiar a los adultos al iniciarse los programas de inmunización pediátrica con este tipo de vacunas. Este efecto, así como los análisis de costo-eficacia, son dependientes de la cobertura en la población vacunada.

\section{¿Qué podemos esperar del estudio clínico de eficacia para PCV-13 en adultos (CAPITA)?}

Este estudio revelará cuál es la eficacia clínica de PCV13 en adultos no vacunados previamente con PPSV-23. Los resultados preliminares fueron presentados en un congreso internacional recientemente (marzo 2014), pero no han sido publicados. Según lo informado, con una población estudiada de aproximadamente 85.000 pacientes, PCV-13 logró reducción de 45,5\% en el primer episodio de neumonía y de $45,0 \%$ para neumonías no bacteriémicas, en ambos casos por serotipos contenidos en la vacuna, además de $75 \%$ de reducción en ENI por estos mismos serotipos.

Sin embargo, este estudio no responderá una pregunta fundamental, como es la eficacia comparativa de PCV-13 versus PPSV-23, porque compara PCV-13 con placebo. Además el estudio se inició a sólo dos años del inicio de vacunación pediátrica universal con la vacuna conjugada heptavalente (PCV-7) en el país donde se desarrolló el protocolo, la cual se mantuvo hasta el año 2011 cuando fue sustituida por PCV-10. Este hecho podría hacer no completamente extrapolables los resultados a poblaciones que han incorporado por varios años las vacunas conjugadas en niños, ya que los cambios epidemiológicos de serotipos implicados en adultos pueden verse en tiempos más prolongados.

\section{¿Cómo es la cobertura de ambas vacunas para los serotipos causales de enfermedad neumocóccica invasora de adultos en Chile?}

Según estudios del Instituto de Salud Pública-ISP, con datos de los serotipos del año 2011 (220 cepas), la cobertura de PCV-13 sería $65 \%$ y la de PPSV-23 de $81 \%$ en adultos mayores de 50 años. Sin embargo, dado que el uso de PCV en población pediátrica de otros países ha significado cambios en la prevalencia de serotipos vaccinales y no vaccinales tanto en niños como en adultos, es posible que en el futuro las coberturas de ambas vacunas varíe, ya que sólo en el año 2011 se introdujo la vacunación infantil programática en el Programa Nacional de Inmunizaciones (PNI) con PCV-10.

\section{¿Cuáles son las recomendaciones actuales para el uso de estas vacunas en adultos?}

Las recomendaciones varían según las distintas sociedades científicas y los gobiernos. El Ministerio de Salud de Chile sólo contempla la vacunación de adultos sobre 65 años de edad, con PPSV-23. En cambio, la Sociedad de Infectología de E.U.A., recomienda el uso de PPSV-23 en todos los adultos de 65 y más años, así como también en los pacientes bajo 65 años que posean algún factor de riesgo como enfermedades pulmonares, cardiovasculares, renales y hepáticas crónicas, diabetes mellitus, inmunodeficiencias, asplenia, fístulas de LCR, implantes cocleares, etilismo, tabaquismo y en residentes de hogares de ancianos. Además recomiendan el uso de PCV-13 en aquellos pacientes mayores de 19 años con condiciones de riesgo que incluyen enfermedades asociadas a inmunosupresión (ej.: onco-hematología, receptores de trasplantes, enfermedades renales crónicas), asplenia, implantes cocleares y fístulas de LCR.

\section{¿Se pueden administrar ambas vacunas en adultos?}

Sí se puede, y si existe indicación para ambas, se administra primero PCV-13 y después de dos meses se puede administrar PPSV-23. En el caso que se haya administrado PPSV-23 y se requiera administrar PCV-13, ésta se debe colocar en un plazo no inferior a un año después, para evitar el fenómeno de disminución en la respuesta.

\section{¿Existe cobertura del sistema de salud pública para vacunas neumocóccicas en adultos?}

El PNI contempla la administración de una dosis de PPSV-23 a todos los adultos de 65 y más años en Chile desde el año 2010.

\section{Resumen}

La enfermedad neumocóccica afecta a una alta proporción de la población, especialmente adultos mayores 
y pacientes con algún grado de inmunosupresión, motivo por el cual las estrategias de prevención como el uso de vacunas, es importante en este grupo de pacientes. Existen dos tipos de vacunas aprobadas para su uso en adultos, po- lisacárida y conjugada, ambas inactivadas, que difieren en su composición e inmunogenicidad. Dado que han surgido nuevos estudios de la vacuna conjugada, recientemente se han modificado las recomendaciones para su uso en adultos.

\section{Referencias bibliográficas}

1.- Mathers CD1, Boerma T, Ma Fat D. Global and regional causes of death. Br Med Bull 2009; 92 : 7-32.

2.- Valdivia C G. Epidemiology of communityacquired pneumonia in adults. Rev Chilena Infectol 2005; 22 Suppl 1: S11-7.

3.- Kyaw M H, Rose C E Jr, Fry A M, Singleton J A, Moore Z, Zell E R, et al. The influence of chronic illnesses on the incidence of invasive pneumococcal disease in adults. J Infect Dis 2005; 192: 377-86.

4.- Vila-Corcoles A, Ochoa Gondar O. Preventing pneumococcal disease in the elderly recent advances in vaccines and implications for clinical practice. Drugs Aging 2013; 30: 263-76.

5.- Vila-Corcoles A, Ochoa Gondar O, Rodríguez-Blanco T. Effectiveness of the 23-valent pneumococcal polysaccharide vaccine against community-acquired pneumonia in the general population aged $\geq 60$ years: 3 years of follow-up in the CAPAMIS study. Clin Infect Dis 2014; 58 (7): 909-17.

6.- Grabenstein J D, Manoff S B. Pneumococcal polysaccharide 23-valent vaccine: Longterm persistence of circulating antibody and immunogenicity and safety after revaccination in adults. Vaccine 2012; 30: 4435-44.

7.- Fedson D S, Nicolas-Spony L, Klemets P, van der Linden M, Marques A, Salleras L, et al. Pneumococcal polysaccharide vaccination for adults: new perspectives for Europe. Expert Rev Vaccines 2011; 10 (8): 1143-67.

8.- Moberley S L, Holden J, Tatham D P, Andrews R M. Vaccines for preventing pneumococcal infection in adults. Cochrane Database Syst Rev 2013 Jan 31; 1: CD000422.

9.- Jiang Y, Gauthier A, Annemans L, van der Linden M, Nicolas-Spony L,
Bresse X. A public health and budget impact analysis of vaccinating at-risk adults and the elderly against pneumococcal diseases in Germany. Expert Rev Pharmacoecon Outcomes Res. 2012; 12 (5): 631-43.

10.- Sanford M. Pneumococcal polysaccharide conjugate vaccine (13-valent, adsorbed) in older adults. Drugs 2012; 72 (9): 1243-55.

11.- http://www.pfizer.com/news/press-release/ press-release-detail/pfizer_presents_detailed_ results_from_landmark_community_acquired_ pneumonia_immunization_trial_in_adults capita_evaluating_efficacy_of_prevenar_13.

12.- Centers for Disease Control and Prevention (CDC). Use of 13-valent pneumococcal conjugate vaccine and 23 -valent pneumococcal polysaccharide vaccine for adults with immunocompromising conditions: Recommendations of the Advisory Committee on Immunization Practices (ACIP). Morb Mortal Wkly Rep 2012; 61 (40): 816-9. 